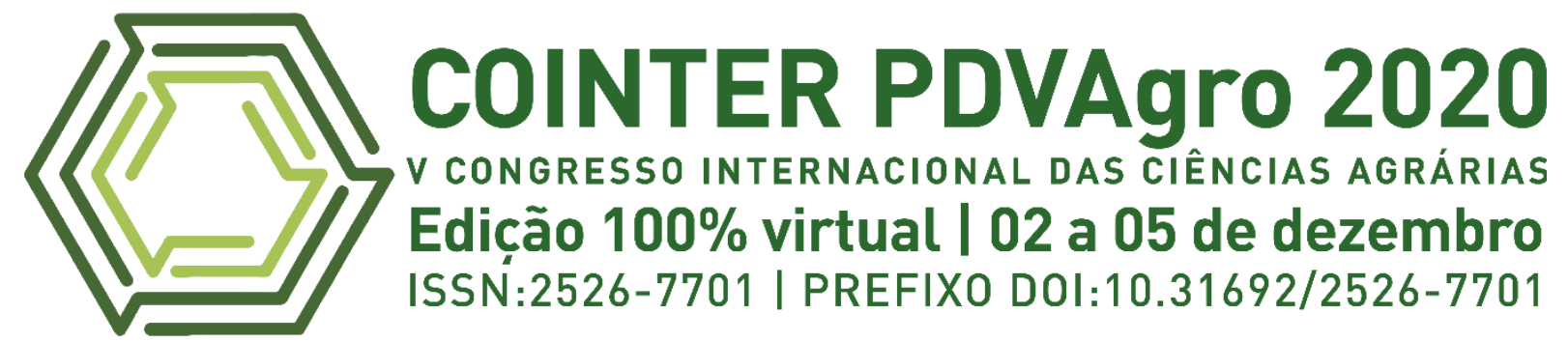

\title{
FIBRAS DIETÉTICAS PARA UMA POSSÍVEL REFORMULAÇÃO DE PRODUTOS CÁRNEOS COM REDUZIDO TEOR DE FOSFATO: UMA REVISÃO
}

\section{FIBRAS DIETÉTICAS PARA UNA POSIBLE REFORMULACIÓN DE PRODUCTOS CÁRNICOS CON CONTENIDO REDUCIDO EN FOSFATO: UNA REVISIÓN}

\section{DIETARY FIBERS FOR A POSSIBLE REFORMULATION OF MEAT PRODUCTS WITH REDUCED PHOSPHATE CONTENT: A REVIEW}

\author{
Apresentação: Comunicação Oral \\ Pinton, M. B. ${ }^{1}$; Leães, Y.S.V. ${ }^{2}$; Pereira, L.C. ${ }^{3}$; Cichoski, A.J. ${ }^{4}$; Campagnol, P.C.B. ${ }^{5}$
}

DOI: https://doi.org/10.31692/2526-7701.VCOINTERPDVAgro.0517

\begin{abstract}
RESUMO
A demanda por produtos cárneos com teor reduzido de aditivos é crescente, devido aos riscos estes apresentam à saúde devido ao consumo elevado. Desta forma, a indústria tem o desafio de encontrar alternativas de redução desses aditivos, promovendo a saudabilidade destes sem afetar a qualidade e aceitabilidade. Uma das alternativas que vem sendo estudadas é a utilização de fibras, como forma de reduzir o teor de fosfato em produtos cárneos. Estudos indicaram que ela possui ação sobre a capacidade de retenção de água (CRA), o que implica diretamente no rendimento do produto (sento esta a principal função do fosfato). Além disso, as fibras dietéticas são prebióticas, o que confere características funcionais e aumenta a saudabilidade do produto. Desta forma, essa revisão objetivou elucidar sobre os diversos tipos de fibras que vem sendo estudadas como uma possível alternativa na redução de fosfatos em produtos cárneos, devido aos efeitos funcionais e tecnológicos que elas proporcionam. Através da fundamentação teórica, observou-se que os fosfatos possuem um papel importante nas características tecnológicas dos produtos cárneos, especialmente em relação à CRA, e que as alternativas vão desde a substituição parcial por outros ingredientes ou aplicação de tecnologias como o ultrassom. As fibras dietéticas são utilizadas como substituição parcial e podem ser de várias origens - cereais, frutas ou hortaliças e, além de atuarem contribuindo para a saúde, apresentam ação quanto a retenção de água, textura e outras características tecnológicas ao serem incorporadas no processamento de alimentos. Para a realização desta revisão foram utilizadas plataformas de periódicos conhecidas (Science Direct, SpringerLink e Publimed, considerando publicações entre 2011-2021. Acerca da ação das fibras

1 Doutoranda em Ciência e Tecnologia dos Alimentos, Universidade Federal de Santa Maria, mbpinton@gmail.com

2 Doutoranda em Ciência e Tecnologia dos Alimentos, Universidade Federal de Santa Maria, yasmimsvl@hotmail.com

${ }^{3}$ Graduanda em Tecnologia dos Alimentos, Universidade Federal de Santa Maria, pereiracorreal@gmail.com

4 Doutor em Tecnologia de Alimentos (UFPR), Professor da Universidade Federal de Santa Maria, cijoale@gmail.com

5 Doutor em Tecnologia de Alimentos (UNICAMP), Professor da Universidade Federal de Santa Maria, paulocampagnol@gmail.com
\end{abstract}


dietéticas especificamente nos produtos cárneos, diversos trabalhos apontaram influência delas sobre o $\mathrm{pH}$ (o qual aumentou ou diminuiu de acordo com a fonte da fibra) e consequentemente na CRA, textura e também na oxidação do produto (o que também aumentou ou reduziu de acordo com a fonte da fibra). Assim, conclui-se que a utilização de fibras dietéticas pode ser viável na substituição de fosfatos em produtos cárneos devido as melhorias de atributos tecnológicos, o que o torna um ingrediente adequado para uso em produtos cárneos. $\mathrm{O}$ aumento do teor de fibra também oferece benefício nutricional para o consumidor e ainda enriquece o produto. Entretanto, recomenda-se a realização de mais estudos para futuras aplicações em uma ampla gama de produtos.

Palavras-Chave: fibra dietética, redução de fosfato, produto cárneo.

\section{RESUMEN}

Crece la demanda de productos cárnicos con contenido reducido de aditivos, debido a los riesgos que presentan para la salud por su elevado consumo. Así, la industria enfrenta el desafío de encontrar alternativas para reducir estos aditivos, promoviendo su salud sin afectar la calidad y aceptabilidad. Una de las alternativas que se ha estudiado es el uso de fibras, como forma de reducir el contenido de fosfato en los productos cárnicos. Los estudios han indicado que tiene una acción sobre la capacidad de retención de agua (CRA), lo que afecta directamente el rendimiento del producto (esta es la función principal del fosfato). Además, las fibras dietéticas son prebióticas, lo que aporta características funcionales y aumenta la salubridad del producto. Así, esta revisión tuvo como objetivo dilucidar los diferentes tipos de fibras que se han estudiado como posible alternativa en la reducción de fosfatos en productos cárnicos, debido a los efectos funcionales y tecnológicos que brindan. A través del fundamento teórico, se observó que los fosfatos juegan un papel importante en las características tecnológicas de los productos cárnicos, especialmente en relación al CRA, y que las alternativas van desde el reemplazo parcial con otros ingredientes o la aplicación de tecnologías como el ultrasonido. Las fibras dietéticas se utilizan como una sustitución parcial y pueden ser de diversos orígenes - cereales, frutas o verduras y, además de actuar para contribuir a la salud, presentan acción sobre la retención de agua, textura y otras características tecnológicas cuando se incorporan al procesamiento de alimentos. Para realizar esta revisión se utilizaron plataformas de revistas conocidas (Science Direct, SpringerLink y Publimed, considerando publicaciones entre 2011-2021). En cuanto a la acción de las fibras dietéticas específicamente en los productos cárnicos, varios estudios señalaron su influencia en el pH (que aumentaba o disminuyó según la fuente de fibra) y consecuentemente en el CRA, textura y también en la oxidación del producto (que también aumentó o disminuyó según la fuente de fibra). Así, se concluye que el uso de fibras dietéticas puede ser viable en la sustitución de fosfatos en productos cárnicos debido a las mejoras en los atributos tecnológicos, lo que lo convierte en un ingrediente adecuado para su uso en productos cárnicos. El aumento del contenido de fibra también ofrece un beneficio nutricional al consumidor e incluso enriquece el producto. se realizan más estudios para futuras aplicaciones en una amplia gama de productos.

Palabras Clave: fibra dietética, reducción de fosfato, productos cárnicos

\section{ABSTRACT}

The demand for meat products with reduced content of additives is growing, due to the risks they present to health due to high consumption. Thus, the industry faces the challenge of finding alternatives to reduce these additives, promoting their health without affecting the quality and acceptability. One of the alternatives that has been studied is the use of fibers, as a way to reduce the phosphate content in meat products. Studies have indicated that it has an action on the water holding capacity (CRA), which directly affects the yield of the product (this is the main function of phosphate). In addition, dietary fibers are prebiotic, which provides functional characteristics and increases the healthiness of the product. Thus, this review aimed to elucidate the different types of fibers that have been studied as a possible alternative in reducing phosphates in meat products, due to the functional and technological effects they provide. Through the theoretical foundation, it was observed that phosphates play an important role in the technological characteristics of meat products, especially in relation to CRA, and that the alternatives range from partial replacement with other ingredients or application of technologies such as ultrasound. Dietary fibers are used as a partial substitution and can be of various origins - cereals, 
fruits or vegetables and, in addition to acting to contribute to health, they present action on water retention, texture and other technological characteristics when incorporated into food processing. To carry out this review, platforms of known journals (Science Direct, SpringerLink and Publimed, considering publications between 2011-2021, were used. Regarding the action of dietary fibers specifically in meat products, several studies pointed out their influence on $\mathrm{pH}$ (which increased or decreased according to the fiber source) and consequently in the CRA, texture and also in the oxidation of the product (which also increased or decreased according to the fiber source). Thus, it is concluded that the use of dietary fibers can be viable in the replacement of phosphates in meat products due to improvements in technological attributes, which makes it a suitable ingredient for use in meat products. The increase in fiber content also offers nutritional benefit to the consumer and even enriches the product. further studies are carried out for future applications in a wide range of products.

Keywords: dietary fiber, phosphate reduction, meat product.

\section{INTRODUÇÃO}

A indústria cárnea deseja mudar a percepção dos produtos cárneos, que é de não saudáveis, desenvolvendo produtos melhorados nutricionalmente através da reformulação por adição de ingredientes funcionais de maior saudabilidade, tais como as fibras, proteínas não cárneas, ou ainda, utilizando tecnologias verdes. Tais produtos podem ser reformulados pela redução do teor de compostos considerados prejudiciais à saúde por alguns consumidores, como por exemplo, gordura, colesterol, aditivos como cloreto de sódio, fosfato e nitrito, bem como pelo aumento do teor de compostos considerados benéficos à saúde humana.

Ambas estratégias devem considerar que os produtos cárneos reformulados devem atender às demandas dos consumidores em relação à qualidade dos produtos cárneos tradicionais, a fim de garantir a aceitabilidade do mesmo. Entretanto, não é uma questão simples devido às funcionalidades tecnológicas que os aditivos possuem na fabricação dos produtos cárneos. Os fosfatos, por exemplo, são aditivos sintéticos amplamente utilizados devido às propriedades tecnológicas atribuidas a ele, como atuar sinergicamente com o cloreto de sódio na extração e solubilização de proteínas miofibrilares, as quais são responsáveis pela capacidade de retenção de água, emulsificação e retenção de gordura. Os fosfatos também aumentam a estabilidade oxidativa e melhoram a maciez e suculência dos produtos cárneos (DYKES et al., 2019).

Nesse contexto de redução de fostato, a utilização de polissacarídeos vem sendo bastante explorada, uma vez que, quando na forma de fibras, possuem características dietéticas e prebióticas que conferem saudabilidade. Com comportamento físico-químico específico, esses polissacarídeos podem ser utilizados para melhorar o perfil nutricional e tecnológico dos produtos cárneos, uma vez que podem atuar aumentando a capacidade de retenção de água,

finalidade primaria do fosfato (KAUR; SHARMA, 2019). Diante do exposto, essa revisão 


\section{FIBRAS DIETÉTICAS PARA UMA POSSÍVEL REFORMULAÇÃO}

objetivou elucidar sobre os diversos tipos de fibras que vem sendo estudadas como uma possível alternativa na redução de fosfatos em produtos cárneos, devido aos efeitos funcionais e tecnológicos que elas proporcionam.

\section{FUNDAMENTAÇÃO TEÓRICA}

Os fosfatos são aditivos muito utilizados na fabricação de produtos cárneos. Atuam sinergicamente com o $\mathrm{NaCl}$ na extração e solubilização de proteínas miofibrilares. Possuem diversas funções como estabilizar o pH, aumentar a capacidade de retenção de água, diminuir a perda de cozimento, melhorar a textura e qualidades sensorais, além de retenção de gordura em emulsões cárneas e dar estabilidade na geleificação formada durante o cozimento (DYKES et al., 2019). O uso de fosfatos em alimentos processados é em função da suas características únicas, uma vez que, melhoram a qualidade do produto. Por isso, sua redução é considerada um grande desafio, podendo haver impactos negativos nas características tecnológicas e sensoriais desses produtos.

Alimentos processados e fast foods são ricos em fosfatos. A alta ingestão deste aditivo pode causar um risco evitável para a saúde. Este aditivo pode causar diversos danos no sistema cardiovascular, insuficiência renal crônica e calcificações cardiovasculares (RITZ et al., 2012).

A tendência de rótulo limpo esta relacionada há mudanças nos padrões alimentares da nossa sociedade, pois muitos produtos alimentícios contém menos aditivos inorgânicos (ASIOLI et al., 2017). Os consumidores estão cada vez mais conscientes da relação entre alimento e saúde. Mas, produtos cárneos ainda são frequentemente percebidos como prejudiciais à saúde devido a sua composição (CAMARA et al., 2020). Os produtos reformulados ou chamados de funcionais adquiriram um enorme interesse como candidatos de sucesso atendendo às percepções dos consumidores de alimentos mais saudáveis (KAUR; SHARMA, 2019).

Já existem diversos estudos com tentativas para redução de fosfato em produtos cárneos através de substituição por outros ingredientes (CHOI et al., 2014; JARVIN et al., 2015; RESCONI et al., 2016; CHOE et al., 2018), redução sem adição de qualquer ingrediente funcional (GLORIEUX et al., 2017) e, recentememente, Pinton et al. (2019) reduziram fosfatos em emulsão cárnea através da utilização de ultrassom. Outros estudos relacionados ao emprego de outras tecnologias emergentes para redução de aditivos também já estão disponiveis (O’FlYNN et al., 2014; BHAT et al., 2020).

É sabido que os produtos cárneos carecem de fibra alimentar, por isso a fibra pode ser incorporada para tornar esses produtos mais saudáveis (KAUR; SHARMA, 2019). A redução 
PINTON et al.

do fosfato e a adição de fibras são abordagens que possuem potencial para melhorar a percepção e o perfil de saúde dos produtos cárneos.

Ingredientes a base de carboidratos, como amidos e fibras dietéticas colaboram na redução de perda de cozimento e melhoram a textura (HENNING et al., 2016). As propriedades e funções das fibras dietéticas dependem da origem da planta, parte botânica utilizada para extrair as fibras, estado físico das partículas de fibra e a tecnologia utilizada para extrair as fibras. As fibras dietéticas têm propriedades multifuncionais: aumentam a capacidade de retenção de água, modulam a textura, estabilizam a gordura em produtos emulsionados e ainda, podem ser exploradas como forma de enriquecimento nutricional em carnes processadas (PIETRACCI et al., 2013).

As fibras dietéticas compreendem um grande grupo de carboidratos com características físico-químicas variadas. Geralmente, são categorizados em duas classes, como fibras dietéticas solúveis, incluindo inulina ou beta-glucano e, fibras dietéticas insolúveis, como a celulose, que é um carboidrato estrutural não utilizado para energia, mas principlamente constitui materiais da parede celular em células vegetais (PRASAD; BONDY, 2019). As fibras são uma mistura complexa de polissacarídeos, as quais incluem celulose, polissacarideos não celulósicos, como mucilagens, substâncias péctinicas, hemiceluloses e componentes não carboidratos como ligninas e gomas (SHARMA et al., 2016). De acordo com a Codex Alimentarius Commission, "fibra dietéticas são definidas como os polímeros de carboidratos com três ou mais de três monômeros, que não são digeridos ou absorvidos no intestino delgado humano" (CODEX ALIMENTARIUS COMMISSION, 2019).

As fibras estão presentes na maioria das frutas, cereais e vegetais, a quantidade e a composição diferem de um alimento para outro. A inclusão de fibras dietéticas na dieta reduz a ocorrencia de doenças, uma vez que a alta ingestão de fibras está associada a um risco reduzido de doenças cardiovasculares, diabetes e certos tipos de câncer (JANZI et al., 2020). A Organização Mundial da Saúde (OMS) recomenda o consumo de $25 \mathrm{~g}$ de fibra dietética por dia, sendo que esta ingestão está relacionada ao consumo de várias fontes alimentares como cereais, feijão, frutas e vegetais (WHO, 2003). As fibras dietéticas afetam a saciedade por meio de diferentes mecanismos, aumentam o tempo de mastigação, levando a um aumento do tempo de exposição dos alimentos na boca, o que aumenta a distensão gástrica, levando a um esvaziamento gástrico retardado e alterando a concentração de hormônios intestinais (SLAVIN; GREEN, 2007). Portanto, o consumo regular de fibras está associado a diversos benefícios como aumento do trânsito intestinal, prevenção/tratamento de diabetes, doenças cardiovasculares, câncer de cólon, doença coronária, redução dos níveis de colesterol no sangue 


\section{FIBRAS DIETÉTICAS PARA UMA POSSÍVEL REFORMULAÇÃO}

e prevenção da obesidade (SERA et al., 2005).

Do ponto de vista industrial, além das propriedades para a saúde, as fibras dietéticas adquirem um lugar específico por suas características funcionais nas diversas formulações de alimentos (KAUR E SHARMA, 2019). Alguns autores utilizam fibras para a redução de gordura em produtos cárneos (CHOI et al., 2010; PIETRASIK; JANZ, 2010), para aumentar o rendimento do cozimento e melhorar a textura (COFRADES, HUGHES, TROY, 2000). Ahmad e Kallem (2018) reportaram a aplicabilidade de beta-glucano como espessantes, estabilizantes, emulsificantes, agentes texturais e também agentes geleificantes, o qual pode ser utilizado na incorporação em produtos lácteos, produtos de panificação e ainda produtos cárneos. Os principais polissacarídeos de cereais e seus papeis em produtos cárneos são bem discutidos no artigo de Kaur e Sharma (2019).

\section{METODOLOGIA}

Para o levantamento dos artigos na literatura, realizou-se uma busca nas seguintes bases de dados: plataforma periódicos da CAPES, ScienceDirect, SpringerLink e Pubmed. A busca foi realizada utilizando termos como "cereal for phosphate reduction in meat product", "phosphate replacer", "water holding capacity”, "functional characteristics". Os critérios de inclusão definidos para a seleção dos artigos foi: artigos publicados em periódicos em inglês. Artigos na íntegra que retratassem a temática referente à revisão nos referidos bancos de dados nos últimos dez anos. Foram utilizados desde revisões de literatura a artigos científicos com a utilização de fibras dietéticas para a redução de fosfatos, gordura e utilização de fibras para produtos mais saudáveis, entre o periodo de 2011 a 2021. Ao total, foram revisados quarenta e cinco artigos científicos, sete artigos de revisão e quatro capítulos de livros.

\section{RESULTADOS E DISCUSSÃO}

A adição de fibras dietéticas, tanto de frutas como de grãos, em produtos cárneos geralmente aumenta a capacidade de retenção de água da matriz alimentar e, consequentemente, o teor de umidade (ELLEUCH et al., 2010). Guedes-Oliveira et al. (2016) utilizaram fibra de caju para redução de gordura em hambúrgueres de frango. Neste estudo, a substituição de gordura afetou positivamente o rendimento de cozimento das amostras, sendo que a amostra com $90 \%$ de fibra de caju apresentou rendimento superior $(88,39 \%)$ quando comparada ao controle $(84,89 \%)$. Outros autores também documentaram melhor rendimento de cozimento em hambúgueres fabricados com fibra de cajú (PINHO et al., 2011). Azevedo e Campagnol (2014) 
PINTON et al.

também constataram um aumento significativo no rendimento total e na retenção de umidade de hambúrgures formulados com farinha de semente de mamão (Carica papaya), de acordo com o aumento da concentração de farinha utilizada.

López-Vargas et al. (2014) notaram que a adição do albedo de maracujá afetou as características de cozimento dos hambúrgueres. Em comparação com a amostra controle, os hambúrgueres reformulados mostraram um maior rendimento de cozimento. Os hambúrgueres reformulados com albedo de maracujá, tiveram menor redução de diâmetro. Nos hambúrgueres reformulados com subproduto de abacaxi de Selani et al. (2016) também foi observada menor perda de cozimento, e consequentemente, maiores valores de retenção de água e gordura, em comparação com a amostra controle. Esses resultados vêm da adição de subprodutos de frutas ricos em fibras, que apresentam maior propriedade de reter água e gordura, reduzindo a perda de exsudados.

Powell et al. (2019) avaliaram o efeito da substituição de fosfatos por fibra citrica em mortadela ao longo de 98 dias de armazenamento. A utilização de $0,75 \%$ de fibra cítrica reduziu a perda de água e gordura, ocasionando em uma melhor estabilidade de emulsão. No entanto, não foram observadas diferenças significativas no rendimento total entre os tratamentos com diferentes concentrações de fibra cítrica e controle. Já Camara et al. (2020), avaliaram o uso de mucilagem de chia em duas formas, em pó e gel, como substituto de fosfato em mortadela bologna. Observou-se menor liberação de líquido e gordura nos tratamento contendo maior conteúdo de mucilagem de chia $(4 \%)$ em relação aos tratamentos contendo apenas $2 \%$, independente da forma de mucilagem utilizada (pó ou gel). De acordo com a forma de incorporação da mucilagem no produto, a formulação com $2 \%$ de mucilagem de chia em gel liberou menor quantidade de liquido, tendo assim, uma melhor estabilidade que a mucilagem de chia em pó.

Pietrasik et al. (2020) observaram que todos os tratamento de hambúrgueres contendo fibras de ervilha tiveram menor perda de cozimento do que aqueles formulados com farelo de trigo ou amido de ervilha. Essa menor perda de cozimento nos hambúrgueres contendo fibra de ervilha foi provavelmente devido à sua capacidade de manter a umidade e a gordura na matriz em comparação com a amostra controle.

O aumento do rendimento devido a capacidade de retenção de água e gordura na matriz cárnea por fibras dietéticas é bem relatada usando diferentes fontes, incluindo película de avelã (TURHAN, SAGIR, USTUN, 2005), fibra de albedo de limão (ALESON-CARBONELL et al., 2005), fibra de noz-tigre (Cyperus esculentus) (SÁNCHEZ-ZAPATA et al., 2009), fibra de abacaxi (HENNING, TSHALIBE, HOFFMAN, 2016), produtos de ameixa seca (JARVIS et 
al., 2012), semente de papoula (GOK et al., 2011), fibra de bagaço de maçã (CHOI et al., 2016), grão de bico e lentilha verde (SHARIATI-IEVARI et al., 2016), aveia (AFSHARI et al., 2015) e farinha de feijão (DZUDIE, SCHER, HARDY, 2002).

Acredita-se que o alto rendimento do cozimento com a adição de ingredientes não cárneos seja devido ao aumento do pH, resultando em maior capacidade de ligação da água e gelificação da proteína durante o tratamento térmico (RUUSUNEN et al., 2003).

No entanto, alguns autores relataram um aumento nos valores de $\mathrm{pH}$ de produtos cárneos adicionados com diferentes tipos de fibras (KTARI et al., 2014; MAGALHÃES et al., 2020). Já Fernandez-Lopez et al. (2019) não encontraram diferença no pH em salsichas reformuladas com diferentes coprodutos de chia. Pietrasik et al. (2020) também não encontraramdiferença no pH de hambúrgueres reformulados com fibra de ervilha. Enquanto que Camara et al. (2020) observaram que o pH das mortadelas bologna contendo chia em pó, sem adição de fosfato, foi menor quando comparado aos demais tratamentos. Já os tratamentos que continham chia e fosfato, apresentaram maiores valores de $\mathrm{pH}$.

A textura dos produtos cárneos cozidos é altamente dependente da geleificação das proteínas miofibrilares. As fibras dietéticas tem demonstrado contribuir para a textura dos produtos cárneos. Fernandez-Lopez et al. (2019) notaram que as mudanças mais evidentes nas propriedades texturais ocorreram quando as salsichas foram adicionadas de farinha de chia, o que resultou em salsichas com menor dureza, mastigabilidade e elasticidade em comparação com a amostra controle. Camara et al. (2020) avaliaram o perfil de textura de salsichas com mucilagem de chia em diferentes dias, e observaram menor firmeza e mastigabilidade aos 60 dias. Em relação à dureza e a elasticidade, observaram menores valores nas amostras contendo mucilagem de chia em pó (2\%) e nas contendo chia em gel (4\%), mostrando que a forma de incorporação da mucilagem, assim como a concentração, interferem neste parâmetro. A coesividade também reduziu pela adição de $4 \%$ de mucilagem de chia em gel.

Powell et al. (2019) observaram que os valores de dureza foram maiores nas amostras com adição de fibra cítrica, quando comparadas com a amostra controle. A coesividade foi menor nos tratamentos com adição de fibra cítrica, em comparação com a amostra controle. De acordo com Pinton et al. (2019) a baixa coesividade e elasticidade representam um sério problema tecnológico que pode afetar as características de qualidade de produtos cárneos emulsionados, como a capacidade de fatiabilidade. Ktari et al. (2014) associa a diminuição da dureza pela diferença na sua composição, resultando em diferentes proporções proteína/gordura/água, o que é uma fator determinante na consistência do gel resultante.

Kim et al. (2014) observou um aumento da dureza, viscosidade e mastigabilidade em 
PINTON et al.

salsichas formuladas com $0,75 \%$ de $\mathrm{NaCl}$ com adição de pó de glasswort (Salicornia herbacea L.). Lopez-Vargas et al. (2014) também observaram aumento nos valores de dureza, viscosidade e mastigabilidade em hambúrgueres com a adição de albedo de maracujá. Outros estudos também relatam que a adição de diferentes fibras, como fibra de celulose, fibra de canade-açúcar, fibra de bagaço de maçã, fibra de aveia, fibra de ervilha, fibra citrica e farelo de centeio resultou em um produto com maior dureza (CHOI et al., 2016; HU et al., 2016; PETERSSON et al., 2014; SONG et al., 2016; ZHAO et al., 2018; ZHUANG et al., 2016).

A oxidação lipídica é um importante determinante da vida útil do produto. Com a utilização de fibra cítrica para redução de fosfato, os valores de TBA nunca foram superiores a $0,2 \mathrm{mg} / \mathrm{kg}$ de malonaldeido, durante todo o período de armazenamento (98 dias), o que sugere que a fibra cítrica não teve efeito pró-oxidante (POWELL et al., 2019). Fernandez-Lopez et al. (2019) relataram que no dia 0 , as salsichas contendo farinha de chia apresentaram maior valor de TBARS. O conteúdo de chia em ácidos graxos altamente insaturados pode ter contribuído para o maior grau de oxidação lipídica observado quando a chia foi adicionada como farinha. Alguns autores relatam que a utilização de fibras em produtos cárneos contribui para o aumento da estabilidade oxidativa (HU et al., 2016, CHOE et al., 2018). Por outro lado, Kim et al. (2016) notou que a adição de fibra insolúvel de casca de soja afetou negativamente na oxidação lipídica de hambúrgures. Já Kumar et al. (2013), relataram que a farinha de casca de soja pode prevenir a oxidação lipídica em nuggets de frango. Do ponto de vista tecnológico, o uso de fibras vegetais de diferentes origens botânicas para desenvolver a qualidade dos produtos cárneos é uma tendência promissora. As fibras têm propriedades multifuncionais e podem ser exploradas como forma de enriquecimento nutricional em carnes processadas (PETRACCI et al., 2013).

\section{CONCLUSÕES}

São expressivos os resultados quanto a utilização de fibras dietéticas como substituintes parciais de fosfatos em produtos cárneos. Diversos estudos relacionaram as fibras dietéticas ao aumento da capacidade de retenção de água e consequentemente, na melhoria de parâmetros tecnológicos, o que as tornam um ingredientes adequados para uso em produtos cárneos. Além disso, aumento do teor de fibra também oferece benefício nutricional para o consumidor e ainda enriquece o produto. Entretanto, recomenda-se a realização de mais estudos objetivando futuras aplicações em uma gama maior de produtos cárneos, avaliando também a influência sobre a conservação e características sensoriais.

\section{REFERÊNCIAS}


AFSHARI, R.; HOSSEINI, H.; KHAKSAR, R.; MOHAMMADIFAR, M. A.; AMIRI, Z.; KOMEII, R.; KHANEGHAH, A.M. Investigation of the Effects of Inulin and $\beta$-Glucan on the Physical and Sensory Properties of Low-Fat Beef Burgers Containing Vegetable Oils: Optimisation of the Formulation Using D-Optimal Mixture Design. Food Technology and Biotechnology, v.53, p.436-445, 2015.

AHMAD, A.; KALLEM, M. Chapter $11 \beta$-Glucan as a Food Ingredient. In Biopolymers for Food Design, p. 315-381, 2018.

ALESON-CARBONELL, L.; FERNÁNDEZ-LÓPEZ, J.; PÉREZ-ALVAREZ, J. A.; KURI, V. Characteristics of beef burger as influenced by various types of lemon albedo. Innovative Food Science \& Emerging Technologies, v.6, p.247-255, 2005.

ASIOLI, D.; ASCHEMANN-WITZEL, J.; CAPUTO, V.; VECCHIO, R.; ANNUNZIATA, A.; NÆS, T.; VARELA, P. Making sense of the "clean label" trends: A review of consumer food choice behavior and discussion of industry implications. Food Research International, v.99, p.58-71, 2017.

AZEVEDO, L.A.; CAMPAGNOL, P.C.B. Papaya seed flour (Carica papaya) affects the technological and sensory quality of hamburgers. International Food Research Journal, v.21, p.2141-2145, 2014.

BHAT, Z.F.; MORTON, J.D.; MASON, S.L.; BEKHIT, A.E.D.A. The application of pulsed electric field as a sodium reducing strategy for meat products. Food Chemistry, v. 306, 125622, 2020 .

CAMARA, A.K.F.I.; VIDAL, V.A.; SANTOS, M.; BERNARDINELLI, O.D.; SABADINI, E.; POLLONIOM M.A.R. Reducing phosphate in emulsified meat products by adding chia (Salvia hispanica L.) mucilage in powder or gel format: A clean label technological strategy. Meat Science, v.163, 108085, 2020.

CHOE, J.; LEE, J.; JO, K.; JO, C.; SONG, M.; JUNG, S. Application of winter mushroom poder as an alternative to phosphates in emulsion-type sausages. Meat Science, v. 143, p. 114 $-118,2018$.

CHOI, J. S.; LEE, H. J.; JIN, S. K.; LEE, H. L.; CHOI, Y. Effect of oyster shell calcium power on the quality of restructured pork ham. Korean Journal of Food Science, v. 34, n. 3, p. $372-$ 377, 2014.

CHOI, Y.S.; CHOI, J.H.; HAN, D.J.; KIM, H.Y.; LEE, M.A.; JEONG, J.Y.; CHUNG, H.J.; KIM, C.J. Effects of replacing pork back fat with vegetable oils and rice bran fiber on the quality of reduced-fat frankfurters. Meat Science, v.84, v.557-563, 2010.

CHOI, Y.S.; KIM, Y.B.; HWANG, K.E.; SONG, D.H.; HAM, Y.K.; KIM, H.W.; SUNG, J.M.; KIM, C.J. Effect of apple pomace fiber and pork fat levels on quality characteristics of uncured, reduced-fat chicken sausages. Poultry Science, v.95, p.1465-1471, 2016.

CODEX ALIMENTARIUS COMMISSION. Codex alimentarius commission and report of the 30th session of the codex committee on nutrition and foods for special dietary uses. Acesso em: 7 out, 2019, from http://www.codexalimentarius.net/download/report/710/al32_26e.pdf, 2019.

COFRADES, S.; HUGHES, E.; TROY, D. J. Effects of oat fibre and carrageenan on the texture 
of frankfurters formulated with low and high fat. European Food Research and Technology, v.211, p.19-26, 2000.

DYKES, G.A.; COOREY, R.; RAVENSDALE, J.T.; SARJIT, A. Phosphates, in: Encyclopedia of Food Chemistry, p. 218-224, 2019.

DZUDIE, T.; SCHER, J.; HARDY, J. Common bean flour as an extender in beef sausages. Journal of Food Engineering, v.52, p.143-147, 2002.

ELLEUCH, M.; BEDIGIAN, D.; ROISEUX, O.; BESBES, S.; BLECKER, C.; ATTIA, H. Dietary fibre and fibre-rich by-products of food processing: Characterisation, technological functionality and commercial applications: A review. Food Chemistry, v.124, p.411-421, 2011.

FERNANDEZ-LOPEZ, J.; LUCAS-GONZÁLEZ, R.; VIUDA-MARTOS, M.; SAYASBARBERÁ, E.; NAVARRO, C.; HAROS, C.M.; PÉREZ-ÁLVAREZ, J.A. Chia (Salvia hispanica L.) products as ingredients for reformulating frankfurters: Effects on quality properties and shelf-life. Meat Science, v.156, p.139-145, 2019.

GLORIEUX, S.; GOEMAERE, O.; STEEN, L.; FRAEYE, I. Phosphate reduction in emulsified meat products: Impact of phosphate type and dosage on quality characteristics. Food Technol. Biotechnol. v. 55, n. 3, p. 390-397, 2017.

GÖK, V.; AKKAYA, L.; OBUZ, E.; BULUT, S. Effect of ground poppy seed as a fat replacer on meat burgers. Meat Science, v.89, p.400-404, 2011.

GUEDES-OLIVEIRA, J.M.; SALGADO, R.L.; COSTA-LIMA， B.R.C.; GUEDESOLIVEIRA, J.; CONTE-JUNIOR, C.A. Washed cashew apple fiber (Anacardium occidentale L.) as fat replacer in chicken patties. LWT - Food Science and Technology, v.71, p.268-273, 2016.

HENNING, S.S.C.; TSHALIBE, P.; HOFFMAN, L.C. Physico-chemical properties of reduced-fat beef species sausage with pork back fat replaced by pineapple dietary fibres and water. LWT - Food Science and Technology, v. 74, p.92-98, 2016.

HU, H.Y.; PEREIRA, J.; XING, L.J.; HU, Y.Y.; QIAO, C.L.; ZHOU, G.H.; ZHANG, W.G. Effects of regenerated cellulose emulsion on the quality of emulsified sausage. LWT - Food Science and Technology, V.70, P.315-321, 2016.

JANZI, S.; DIAS, J.A.; MARTINSSON, A.; SONESTEDT, E. Association between dietary fiber intake and risk of incident aortic stenosis. Nutrition, Metabolism and Cardiovascular Diseases, in: 10.1016/j.numecd.2020.07.015, 2020.

JARVIS, N. O'BRYAN, C. A.; RICKE S. C.; CRANDALL, P. G. The functionality of plum ingredients in meat products: A review. Meat Science, v. 102, p. 41 - 48, 2015.

JARVIS, N.; CLEMENT, A.R.; O'BRYAN, C.A.; BABU, D.; CRANDALL, P.G.; OWENS, C.M.; MEULLENET, J.F.; RICKE, S.C. Dried Plum Products as a Substitute for Phosphate in Chicken Marinade. Journal of Food Sicence, v. 77, 2012.

KAUR, R.; SHARMA, M. Cereal polysaccharides as sources of functional ingredient for reformulation of meat products: A review. Journal of Functional Foods, v.62, 103527, 2019. 
KIM, H.W.; HWANG, K.E.; SONG, D.H.; KIM, Y.J.; LIM, Y.B.; HAM, Y.K.; YEO, E.J.; CHANG, S.J.; CHOI, Y.S.; KIM, C.J. Effect of glasswort (Salicornia herbacea L.) on the texture of frankfurters. Meat Science, v.97, p.513-517, 2014.

KIM, H.W.; MILLER, D.K.; LEE, Y.J.; KIM, Y.H.B. Effects of soy hull pectin and insoluble fiber on physicochemical and oxidative characteristics of fresh and frozen/thawed beef patties. Meat Science, v.117, p.63-67, 2016.

KTARI, N.; SMAOUI, S.; TRABELSI, I.; NASRI, M.; SALAH, R.B. Chemical composition, techno-functional and sensory properties and effects of three dietary fibers on the quality characteristics of Tunisian beef sausage. Meat Science, v.96, p.521-525, 2014.

KUMAR, V.; BISWAS, A.K.; SAHOO, J.; CHATLI, M.K.; SIVAKUMAR, S. Quality and storability of chicken nuggets formulated with green banana and soybean hulls flours. Journal od Food Science and Technology, v.50, p.1058-1068, 2013.

LÓPEZ-VARGAS, J.H.; FERNÁNDEZ-LÓPEZ, J.; PÉREZ-ÁLVAREZ, J.A.; VIUDAMARTOS, M. Quality characteristics of pork burger added with albedo-fiber powder obtained from yellow passion fruit (Passiflora edulis var. flavicarpa) co-products. Meat Science, v.97, p.270-276, 2014.

MAGAlhães, I. M. C.; PAGLARINI, C. S.; VIDAL, V. A. S.; POLlONIO, M. A. R. Bamboo fiber improves the functional properties of reduced salt and phosphate-free Bologna sausage. Journal of Food Processing and Preservation. In: doi.org/10.1111/jfpp.14929, 2020.

O'FLYNN, C.C.; CRUZ-ROMERO, M.C.; TROY, D.; MULLEN, A.M.; KERRY, J.P. The application of high-pressure treatment in the reduction of phosphate levels in breakfast sausages.Meat Scice, v.96, p.633-639, 2014.

PETERSSON, K.; GODARD, O.; ELIASSON, A.C.; TORNBERG, E. The effects of cereal additives in low-fat sausages and meatballs. Part 2: Rye bran, oat bran and barley fibre. Meat Science, v.96, p.503-508, 2014.

PIETRACCI, M.; BIANCHI, M.; MUDALAL, S.; CAVANI, C. Functional ingredientes for poultry meat products. Trends in Food Science \& Technology, v.33, p.27-39, 2013.

PIETRASIK, Z.; JANZ, J.A.M. Utilization of pea flour, starch-rich and fiber-rich fractions in low fat Bologna. Food Research International, v. 43, p. 602-608, 2010.

PIETRASIK, Z.; SIGVALDSON, M.; SOLADOYER, O.P.; GAUDETTE, N.J. Utilization of pea starch and fibre fractions for replacement of wheat crumb in beef burgers. Meat Science, v.161, 106974, 2020.

PINHO, L.X.; AFONSO, M.R.A.; CARIOCA, J.O.B.; DA COSTA, J.M.C., RAMOS, A.M. The use of cashew apple residue as source of fiber in low fat hamburgers. Ciência e Tecnologia de Alimentos, v.31, p.941-945, 2011.

PINTON, M.B.; CORREA, L.P.; FACCHI, M.M.X.; HECK, R.T.; LEÃES, Y.S.V.; CICHOSKI, A.J.; LORENZO, J.M.; DOS SANTOS, M.; POLLONIO, M.A.R.; CAMPAGNOL, P.C.B. Ultrasound: a new approach to reduce phosphate contente of meat emulsions. Meat Science, v.152, p.88-95, 2019. 
POWELL, M.J.; SEBRANEK, J.G.; PRUSA, K.J.; TARTÉ, R. Evaluation of citrus fiber as a natural replacer of sodium phosphate in alternatively-cured all-pork Bologna sausage. Meat Science, v.157, p.1-7, 2019.

PRASAD, K.N.; BONDY, S.C. Dietary fibers and their fermented short-chain fatty acids in prevention of human diseases. Bioactive Carbohydrates and Dietary Fibre, v.17, 100170, 2019.

RESCONI, V. C.; KEENAN, D. F.; GARCÍA, E.; ALLEN, P.; KERRY, J. P.; HAMILL, R. M. The effects of potato and rice starch as substitutes for phosphate in and degree of comminution on the technological, instrumental and sensory characteristics of restructured ham. Meat Science, v. 121, p. 127 - 134, 2016.

RESCONI, V.C.; KEENAN,D.F.; GOUGH, S.; DORAN,L.; ALLEN, P.; KERRY,J.P.; HAMELL, R. M. Response surface methodology analysis of rice starch and fructooligosaccharides as substitutes for phosphate and dextrose in whole muscle cooked hams. LWT-Food and Science Technology, v. 64, p. 946-958, 2015

RITZ, E.; HAHN, K., KETTELER, M.; KUHLMANN, M.K.; MANN, J. Phosphate Additives in Food a Health Risk. Deutsches Arzteblatt International, v. 109, 10.3238/arztebl.2012.0049, 2012

RUUSUNEN, M. VAINIONPAA, J.; PUOLANNE, E.; LYLY, M.; LAHTEENMAKI, L.; NIEMISTO, M.; AHVENAINEN, R. Physical and sensory properties of low-salt phosphatefree frankfurters composed with various ingredientes. Meat Science, v.63, p.9-16, 2003.

SÁNCHEZ-ZAPATA， E.; FUENTES-ZARAGOZA， E.; FERNÁNDEZ-LÓPEZ, J.; SENDRA, E.; SAYAS, E.; NAVARRO, C.; PÉREZ-ÁLVAREZ, J.A. Preparation of Dietary Fiber Powder from Tiger Nut (Cyperus esculentus) Milk ("Horchata") Byproducts and Its Physicochemical Properties. Journal of Agricultural and Foos Chemistry, v. 57, p.7719-7725, 2009.

SELANI, M.M.; SHIRADO, G.A.N.; MARGIOTTA, G.B.; SALDAÑA, E.; SPADA, F.P.; PIEDADE, S.M.S.; Contreras-Castillo, C.J.; Canniatti-Brazaca, S.G. Effects of pineapple byproduct and canola oil as fat replacers on physicochemical and sensory qualities of low-fat beef burger. Meat Science, v.112, p.69-76, 2016.

SERA, N.; MORITA, K.; NAGASOE, M.; TOKIEDA, H.; KITAURA, T.; TOKIWA, H. Binding effect of polychlorinated compounds and environmental carcinogens on rice bran fiber. The Journal of Nutritional Biochemistry, v.16, p.50-58, 2005.

SHARIATI-IEVARI, S.; RYLAND, D.; EDEL, A.; NICHOLSON, T.; SUH, M.; ALIANI, M. Sensory and Physicochemical Studies of Thermally Micronized Chickpea (Cicer arietinum) and Green Lentil (Lens culinaris) Flours as Binders in Low-Fat Beef Burgers. Journal of Food Sicence, v.81, 2016.

SHARMA, S.K.; BANSAL, S.; MANGAL, M.; DIXIT, A.K.; GUPTA, R.K.; MANGAL, A.K. Utilization of Food Processing By-products as Dietary, Functional, and Novel Fiber: A Review. Journal Critical Reviews in Food Sicence and Nutrition, v.56, p.1647-1661, 2016.

SLAVIN, J.; GREEN, H. Dietary fibre and satiety. Nutrition Bulletin, v,32, in: doi.org/10.1111/j.1467-3010.2007.00603.x, 2007. 
SONG, J.; PAN, J.; WU, J.; REN, F. The improvement effect and mechanism of citrus fiber on the water-binding ability of low-fat frankfurters. Journal of Food Science and Technology, v.53, p.4197-4204, 2016.

TURHAN, S.; SAGIR, I.; USTUN, N.S. Utilization of hazelnut pellicle in low-fat beef burgers. Meat Science, v.71, p.312-316, 2005.

WHO. Diet, nutrition and the prevention of chronic diseases: report of a joint WHO/FAO expert consultation. WHO Technical Report Series 916, 2003.

ZHAO, Y.; HOU, Q.; ZHUANG, X.; WANG, Y.; ZHOU, G.; ZHANG, W. Effect of regenerated cellulose fiber on the physicochemical properties and sensory characteristics of fatreduced emulsified sausage. LWT - Food Science and Technology, v.97, p.157-163, 2018.

ZHUANG, X.; HAN, M.; KANG, Z.; WANG, K.; BAI, Y.; XU, X.; ZHOU, G. Effects of the sugarcane dietary fiber and pre-emulsified sesame oil on low-fat meat batter physicochemical property, texture, and microstructure. Meat Science, v.113, p.107-115, 2016. 